\title{
Coopération éducative entre les États-Unis et le Mexique : quelles perspectives pour les élèves transnationaux?
}

Educational cooperation between the U.S. and Mexico: what prospects for transnational students?

Cooperación educativa entre Estados Unidos y México: ¿qué perspectivas para los estudiantes transnacionales?

\section{Isabelle Sinic}

\section{(2) OpenEdition}

\section{Journals}

Édition électronique

URL : https://journals.openedition.org/ideas/11589

DOI : $10.4000 /$ ideas. 11589

ISSN : 1950-5701

\section{Éditeur}

Institut des Amériques

\section{Référence électronique}

Isabelle Sinic, «Coopération éducative entre les États-Unis et le Mexique : quelles perspectives pour les élèves transnationaux ? », IdeAs [En ligne], 18 | 2021, mis en ligne le 01 octobre 2021, consulté le 21 octobre 2021. URL : http://journals.openedition.org/ideas/11589; DOI : https://doi.org/10.4000/ideas. 11589

Ce document a été généré automatiquement le 21 octobre 2021.

\section{cc) (1)}

IdeAs - Idées d'Amériques est mis à disposition selon les termes de la licence Creative Commons Attribution - Pas d'Utilisation Commerciale - Pas de Modification 4.0 International. 


\title{
Coopération éducative entre les États-Unis et le Mexique : quelles perspectives pour les élèves transnationaux?
}

\author{
Educational cooperation between the U.S. and Mexico: what prospects for \\ transnational students? \\ Cooperación educativa entre Estados Unidos y México: ¿qué perspectivas para \\ los estudiantes transnacionales?
}

Isabelle Sinic

\section{Introduction}

1 Les enfants et les adolescents sont une population largement sous-étudiée dans les travaux sur les migrations internationales et jusqu'à très récemment totalement absente des études migratoires Mexique / États-Unis. Cette absence tient tout d'abord à l'histoire des mouvements migratoires eux-mêmes puisque tout au long du xxe siècle, la migration du Mexique vers les États-Unis a été largement constituée d'hommes adultes, poussés à l'émigration par la faible croissance de l'économie mexicaine conjuguée au besoin accru de main d'œuvre aux États-Unis (Imaz C., 2007). Jusqu'au milieu des années 1980, cette migration était souvent temporaire et circulaire, l'envoi de devises suffisant à faire vivre les femmes et les enfants restés au pays ${ }^{1}$. À la fin du Xx $x^{e}$ siècle, aux États-Unis, la mise en œuvre de politiques fédérales de restriction des flux migratoires et le durcissement des contrôles à la frontière ont mis fin à cette circularité et contribué à fixer, par la réunification familiale, la diaspora mexicaine aux États-Unis.

2 À partir de 2005 toutefois, les effets combinés de la récession économique des années 2008-2009 et de la pression croissante exercée par les autorités fédérales et locales à l'encontre des sans-papiers ont conduit de nombreuses familles d'origine mexicaine à 
envisager un retour définitif, plus ou moins contraint, vers leur pays d'origine (Hernández-León, R. et V. Zúñiga, 2016 : 174, Escobar Latapí A., et al., 2013 : 17). Ainsi, depuis quelques années, les démographes ont noté une baisse du nombre d'immigrés mexicains présents aux États-Unis et mis en évidence un important mouvement de migration de retour (Giorguli S. et E. Gutiérrez, 2011, Passel J., et al., 2012, GonzálezBarrera A., 2015). Après avoir atteint un pic à 12,5 millions de personnes en 2007, le nombre d'immigrés mexicains a chuté à 10,9 millions en 2019 (Hazán M., $2014: 2$, Israel E. et J. Batalova, 2020). Si le phénomène de migration de retour n'est pas nouveau, puisqu'il faisait partie intégrante du phénomène de migration circulaire, en revanche, la stabilisation de la diaspora mexicaine aux États-Unis depuis les années 1980 et l'allongement de la durée de résidence dans le pays d'accueil ont fondamentalement transformé la composition de la population des migrants de retour ${ }^{2}$. Cette population est désormais constituée de familles à statut mixte comprenant des immigrés de première, deuxième et troisième générations, dont une part importante de mineurs nés aux États-Unis. Ce nouveau déplacement des familles d'origine mexicaine a entraîné une augmentation inédite du nombre de citoyens des États-Unis au Mexique. En 2019, cette population était estimée à 1,5 million de personnes, dont environ 600000 mineurs, ce qui en fait aujourd'hui la population d'émigrés états-uniens la plus importante au monde (U.S. Department of State, 2020, Selee A. et al., 2019 : 11).

3 Ce phénomène rebat aujourd'hui les cartes du débat sur les migrations entre le Mexique et les États-Unis, longtemps envisagé selon une perspective sud-nord uniquement, et conduit à une inversion des préoccupations relatives à l'intégration scolaire des enfants de migrants. En effet, le flux récent des retours vers le Mexique incite les chercheurs et les gouvernements des deux pays à s'interroger sur les opportunités éducatives offertes aux enfants d'origine mexicaine dont les parcours scolaires se distinguent désormais par leur caractère transnational, c'est-à-dire par une scolarisation dans les deux pays, chacun étant, selon les cas, pays de départ ou pays d'arrivée. Ainsi, à la question, toujours pertinente, des besoins éducatifs spécifiques des enfants d'immigrés mexicains scolarisés aux États-Unis s'ajoute désormais celle de l'intégration, voire de la réintégration, de ces enfants par les institutions scolaires mexicaines.

Les travaux empiriques sur cette question sont encore relativement limités. Les pionniers en la matière sont le sociologue Víctor Zúñiga et l'anthropologue Edmund $\mathrm{T}$. Hamann, dont les enquêtes de terrain menées dans les écoles de quatre États du Mexique entre 2004 et $2010^{3}$ ont fourni les premières informations permettant de quantifier cette population d'élèves transnationaux ${ }^{4}$ et de décrire leurs trajectoires scolaires entre les États-Unis et le Mexique (Zúñiga V., et al., 2008a, 2008b, Zúñiga, V. et E. Hamann 2009, 2013, 2020). Les difficultés rencontrées par les élèves transnationaux au Mexique, liées notamment à leur maîtrise limitée de la langue espagnole en contexte scolaire, ont également été documentées par des travaux récents invitant les enseignants à adapter leurs pratiques et les autorités mexicaines à mettre en place des politiques publiques ciblées (Zúñiga V. et al., 2008b, Zúñiga V. et M. Vivas-Romero, 2015, Borjian A., et al., 2016, Jensen B. et al., 2017, Herrera M. et E. Montoya Zavala, 2019).

Cet article procèdera à une revue de la littérature sur le sujet et abordera la coopération qui existe entre les États-Unis et Mexique dans le domaine de l'éducation sous l'angle du transnationalisme des élèves d'origine mexicaine, entendu ici comme la fréquentation successive, et potentiellement répétée, d'établissements scolaires situés 
dans les deux pays (Martinez-León N. et P. Smith, 2003, Zúñiga, V. et E. Hamann, 2009). Il s'agira d'examiner l'histoire des collaborations entre les États-Unis et le Mexique dans le domaine de l'éducation à l'aune des données empiriques collectées par les démographes, anthropologues et sociologues des deux pays. Il conviendra de rappeler d'abord que les mécanismes bilatéraux de coopération éducative ont été mis en place dans le contexte migratoire et économique des années 1980 et 1990, c'est-à-dire à une période de forte migration du Mexique vers les États-Unis. Pour cette raison, ces programmes demeurent centrés sur l'intégration scolaire des élèves mexicains aux États-Unis et ne répondent que partiellement aux besoins des élèves transnationaux issus de la migration de retour des années 2005-2015. À travers l'exemple des initiatives prises par les autorités éducatives de Californie et de Basse-Californie, il s'agira ensuite de démontrer qu'au niveau régional, ces dynamiques démographiques ouvrent la voie à de nouveaux réseaux de coopération transnationale dont la zone frontière entre le Mexique et les États-Unis constitue une zone pionnière.

\section{Aux origines d'une approche bilatérale des questions scolaires : la migration saisonnière des travailleurs mexicains}

6 Aux États-Unis, la première formulation d'une prise en charge spécifique des enfants de migrants apparaît en 1966 sous forme d'un amendement à la loi de réforme de l'enseignement primaire et secondaire, Elementary and Secondary Education Act (ESEA). Le premier Programme pour l'éducation des migrants, Migrant Education Program, voit le jour afin de faciliter la continuité éducative des enfants des familles mexicaines qui se déplacent à l'intérieur des États-Unis au rythme des saisons agricoles. Cette assistance prend la forme de fonds fédéraux distribués aux autorités éducatives des États concernés. Elle sert notamment à la transmission des dossiers administratifs, afin de limiter les ruptures scolaires, et au financement de cours de remise à niveau. Il s'agit à ce moment-là d'une approche strictement nationale qui ne concerne que les migrations internes, mais ce cadre juridique sera par la suite étendu aux migrations internationales entre le Mexique et les États-Unis, et constitue, en cela, les prémisses d'une forme institutionnalisée de coopération.

7 En 1976, les flux importants de travailleurs agricoles migrant entre l'État du Michoacán et la Californie donnèrent l'impulsion aux premiers efforts de collaboration entre les autorités éducatives des deux pays. Un grand nombre d'enfants des écoles du district scolaire unifié de Pajaro Valley à Santa Cruz en Californie étaient originaires du village de Gómez Farías, dans le Michoacán, au Mexique. Ces élèves passaient entre un à six mois à Gómez Farías (généralement de novembre à avril) mais s'y voyaient refuser l'accès aux écoles parce qu'ils manquaient la rentrée scolaire, en septembre de chaque année. Les contacts établis par une enseignante de Pajaro Valley, Arlene Dorn, avec les autorités scolaires de Gómez Farías permirent d'instaurer une coopération à l'échelle locale, dont la première étape consista en l'élaboration d'un document commun permettant le transfert d'informations d'un système scolaire à l'autre. Ce document, le Transfer Document for Binational Migrant Student / Documento de Transferencia del Estudiante Migrante Binacional, contenait la liste des matières suivies, le niveau atteint, ainsi qu'un calendrier récapitulant les périodes scolaires passées dans chaque pays. 
8 En 1982, cette collaboration donna naissance à l'actuel Programme binational pour l'éducation des migrants, Binational Migrant Education Initiative (BMEI) / Programa Binacional de Educación Migrante (PROBEM). Outre la transmission des dossiers scolaires, ce programme permit de financer des séminaires de formation professionnelle, des échanges de postes d'enseignants et un système de prêt de manuels scolaires en langue espagnole (Dolson, D. et G. Villaseñor, 1996 : 128-129). En 1990, les « bénéfices mutuels » d'une coopération éducative entre les deux pays furent formalisés par la signature d'un protocole d'accord réaffirmant la volonté d'améliorer les services éducatifs offerts à la population d'origine mexicaine vivant aux États-Unis (Cavazos L. et M. Bartlett-Diaz, 1990).

9 La signature d'annexes, tous les deux ans, a permis d'adosser les intentions des deux parties à leurs priorités politiques et économiques respectives. Ainsi, le protocole d'accord prit tout son sens lors de la mise en œuvre, à partir de 1994, de l'Accord de libre-échange nord-américain (ALENA). La seconde annexe exprimait en effet l'intention de s'entendre sur une éducation qui contribuerait à fournir une maind'œuvre hautement qualifiée et productive, répondant aux besoins des entreprises situées le long de la frontière entre les deux pays (Varisco de Garcia N. et E. Garcia, 1996 : 156). La neuvième annexe, qui décrit les activités de la période 2012-2014, fait état des nombreux domaines dans lesquels cette coopération s'applique désormais : mobilité internationale des enseignants et des étudiants, partenariats avec le secteur privé, enseignement de la langue et promotion de la culture de chaque pays, renforcement de l'éducation dans le domaine des sciences et des nouvelles technologies. Dans le domaine de l'enseignement primaire et secondaire, ce document fait pour la première fois la distinction entre migrants saisonniers et migrants de retour, et mentionne la nécessité de faciliter l'intégration scolaire des élèves transnationaux dont le niveau de maîtrise de la langue espagnole et la connaissance de la culture mexicaine sont insuffisants (Córdova Villalobos J. et A. Duncan, 2012 : 1-2).

Néanmoins, en dépit de ces ambitions, de nombreux observateurs de l'éducation notent la portée davantage symbolique que réelle de ces engagements et pointent du doigt les failles structurelles de la coopération bilatérale et des politiques éducatives des deux pays, en particulier s'agissant des élèves transnationaux scolarisés au Mexique.

\section{Les élèves transnationaux : une population dans l'angle mort des politiques éducatives États- Unis / Mexique}

11 La première raison permettant d'expliquer la portée limitée du protocole de 1990 sur l'éducation des élèves transnationaux tient à l'architecture même de cette coopération, fondée sur une asymétrie institutionnelle qui fragilise la mise en œuvre et le financement des programmes. Aux États-Unis, les fonds proviennent du département d'État, par le biais de la Commission binationale États-Unis / Mexique, qui alloue ensuite aux autorités éducatives des États fédérés les fonds auxquels ils sont éligibles. Le montant de ces fonds dépend donc fortement de la politique étrangère du gouvernement. Or, les priorités en matière de coopération bilatérale, incarnées par le programme Mérida mis en place en décembre 2008, sont orientées vers la lutte contre le crime organisé et la sécurisation de la frontière, et laissent peu de place aux questions 
d'éducation. Aucune annexe au protocole de 1990 n'a été signée depuis 2012, et les financements fédéraux ont été réduits de manière drastique (Martin-Wenzl M., 2013 : 296, Anderson J., 2016: 21). Au Mexique, l'éducation est une compétence du gouvernement fédéral mais les mesures de décentralisation prises en 1992 ont transféré aux entités fédératives la responsabilité de l'enseignement primaire et secondaire, ainsi que la formation des maîtres (Malgouyres F., 2013: 85). La mise en œuvre et le financement du PROBEM incombent aux États fédérés, ce qui génère de fortes disparités entre les régions. Ainsi, bien que ce programme soit en place depuis 1989 au Mexique, de nombreux élèves n'en bénéficient pas.

Ce hiatus institutionnel se traduit également par un manque de lisibilité du statut juridique des élèves transnationaux, certains étant nés aux États-Unis de parents mexicains, d'autres au Mexique. Lisa Gisvold, responsable des services aux citoyens à l'ambassade des États-Unis à Mexico, résume en ces termes la situation :

Un grand nombre de ces enfants ont passé la majeure partie de leur vie dans l'ombre, tant aux États-Unis qu'au Mexique - d'abord en tant qu'enfants de parents sans papiers aux États-Unis, puis en tant qu'enfants qui ne peuvent prouver leur identité et leur citoyenneté au Mexique ${ }^{5}$. (Lakhani N. et M. Jacobo, 2016)

Les difficultés des familles à faire valoir le statut de leurs enfants en tant que citoyens binationaux ou citoyens mexicains accroissent le risque d'interruption du parcours scolaire et aboutissent dans certains cas à la déscolarisation complète de ces élèves « doublement sans-papiers » (Anderson J., $2016: 15$ ).

Aux États-Unis comme au Mexique, les politiques éducatives continuent à être fondées sur une approche sud-nord des migrations et elles sont, de ce fait, mal adaptées à la situation particulière des élèves transnationaux. Le Mexique, dont l'économie bénéficie des liquidités envoyées par les travailleurs migrants à leurs familles, joue un rôle actif dans l'éducation de sa diaspora aux États-Unis. Par exemple, les partenariats mis en place à la fin des années 1980 par le biais du Programme pour les Communautés Mexicaines à l'Étranger, Programa para las Comunidades Mexicanas en el Extranjero, et le relais important que constituent les agences consulaires, favorisent à différents niveaux de gouvernance et dans différents domaines, éducation des migrants, enseignement bilingue et enseignement supérieur, la réussite scolaire et le rayonnement culturel de la communauté mexicaine aux États-Unis (de la Garza R., 1997 : 85, Laglagaron L., $2010: 28-29$, Tapia J. et C. Dorn, $2019:$ 79). Il n'existe à ce jour aucune mesure comparable émanant du gouvernement des États-Unis à destination des élèves transnationaux présents au Mexique.

Les États-Unis disposent, depuis les années 1970, d'un large éventail de programmes d'enseignement bilingue visant à faciliter l'intégration des élèves non-anglophones. À l'origine, les fonds fédéraux alloués dans le cadre de la loi sur l'enseignement bilingue de 1968, Bilingual Education Act, servaient trois objectifs : l'acquisition de l'anglais par les élèves allophones, l'enseignement des matières de base dans la langue maternelle des apprenants et le maintien de l'identité culturelle des enfants migrants. En 2002, la loi sur l'acquisition de l'anglais, English Language Acquisition Act, a vidé de sa dimension interculturelle et plurilingue la législation initiale. Les programmes aujourd'hui en vigueur reposent essentiellement sur une méthode consistant à « immerger » les élèves allophones dans un enseignement dispensé en anglais. Pour les enfants d'origine mexicaine, cette méthode aboutit à la perte de leur langue maternelle, parlée uniquement dans la sphère familiale. De retour au Mexique, leur maîtrise limitée de 
l'espagnol écrit entrave fortement la poursuite de leur scolarité (Panait C. et V. Zúñiga, 2016 : 239). À cela s'ajoute le fait que, comme le notent Zúñiga et Hamann, la question de l'intégration des élèves anglophones au Mexique est demeurée absente des réformes récentes:

Cette population scolaire n'est pas prise en compte dans les projets et programmes éducatifs du Mexique, ni dans le Plan de développement national 2013-2018, ni dans le Programme sectoriel d'éducation 2013-2018, ni dans le Modèle éducatif 2016, documents qui encadrent la réforme de l'enseignement de $2016^{6}$. (Zúñiga $\mathrm{V}$. et $\mathrm{E}$. Hamann, 2019 : 228)

Dans une étude commandée en 2008 par le secrétariat à l'éducation publique, Zúñiga, Hamann et Sánchez García avaient pointé du doigt, outre l'élaboration au niveau national de programmes de formation des enseignants et de programmes d'éducation interculturelle à même de favoriser l'intégration scolaire des migrants internationaux, l'importance d'aborder les questions d'éducation et de migration selon une approche transnationale reposant sur deux axes complémentaires: un axe sociologique permettant de mettre au jour les raisons de l'échec scolaire des migrants internationaux d'origine mexicaine de part et d'autre de la frontière, et un axe pédagogique visant à promouvoir une éducation biculturelle et bilingue adaptée aux trajectoires migratoires de ces élèves (Zúñiga, Víctor, Edmund T. Hamann et Juan Sánchez García, 2008 : 76-90).

17 Si elle demeure encore limitée dans les politiques de coopération et les politiques éducatives des deux pays, cette dynamique transnationale est aujourd'hui observable dans les politiques publiques conduites au niveau régional, plus particulièrement au sein du vaste espace transfrontalier États-Unis/Mexique. En effet, en dépit d'un contexte politique dominé par les questions d'immigration clandestine, de trafics illicites et de sécurité nationale, le sort des élèves transnationaux cristallise aujourd'hui les enjeux éducatifs et culturels qui marqueront l'avenir des États-Unis et du Mexique en tant que sociétés mondialisées et interdépendantes (Selee, A, 2018: 270). La zone frontière, en tant que point de contact et espace d'interactions, constitue un champ d'observation privilégié des évolutions institutionnelles qui accompagnent ces changements. Plus particulièrement, s'agissant de la question spécifique des élèves transnationaux, les efforts de collaboration déployés par les États de Californie et Basse-Californie montrent que l'école n'a plus seulement pour mission de former les citoyens de la nation et d'intégrer les étrangers, mais elle constitue un nouveau «champ social transnational» (Portes A. et al., 1999: 228) destiné à former des individus bilingues et biculturels, issus de deux sociétés et dont l'avenir se construit désormais de part et d'autre de la frontière :

C'est un espace où les élèves qui ont commencé leur scolarité au Mexique mais qui sont nés aux États-Unis rencontrent des élèves qui sont toujours allés à l'école aux États-Unis mais qui sont mexicains de naissance. Les enfants migrants développent leur expérience et définissent leur identité en traversant les frontières qui délimitent les États nationaux. Face à cette frontière qui divise, les migrants transnationaux découvrent d'autres manières possibles de comprendre cette notion si riche en significations ${ }^{7}$. (Sierra Pérez S. et López López Y., 2013 : 42) 


\section{L'intégration scolaire entre Californie et Basse- Californie : du transnationalisme des communautés à la transnationalisation des réseaux éducatifs}

Les migrants de retour ayant tendance à s'installer dans les zones urbaines où la croissance économique est la plus forte, il n'est pas surprenant de constater que les élèves transnationaux sont surreprésentés dans les grandes métropoles des États frontaliers. Les données publiées en 2015 par l'Institut mexicain de statistiques et de géographie, Instituto Nacional de Estadística y Geografía (INEGI), montrent que les élèves transnationaux représentent plus de 3\% de la population scolaire dans quatre des six États frontaliers mexicains : Basse-Californie (7,2\%), Chihuahua (5,6\%), Sonora (4\%) et Tamaulipas (3,6\%) (Jensen B. et al., $2017: 10){ }^{8}$. Alors que lors les premières enquêtes avaient montré que les deux-tiers des élèves transnationaux étaient nés au Mexique (Zúñiga V., 2012), en 2015, ces enfants sont dans leur très large majorité nés aux ÉtatsUnis (Jensen B. et al., 2017 : 10).

19 À partir de 2005, les agents en charge de l'application du PROBEM en Basse-Californie ont vu le nombre de demandes d'inscription augmenter de manière significative. Ces derniers ont contribué à faire remonter auprès des autorités éducatives des États, outre les difficultés rencontrées par les familles pour faire valoir l'identité et le parcours scolaire antérieur de leurs enfants, les problèmes d'intégration rencontrés par les élèves transnationaux et l'inadaptation du programme fédéral aux évolutions $d u$ contexte migratoire (Sierra Pérez S. et López López Y., 2013 : 40, Osuna García, J.-C. et J. Rabelo Ramírez, 2019).

Cette visibilité nouvelle des élèves transnationaux en Basse-Californie fait aujourd'hui écho aux préoccupations des acteurs de l'éducation qui tentent, depuis plusieurs décennies, d'expliquer les difficultés d'apprentissage des élèves d'origine mexicaine aux États-Unis (Gándara P. et F. Contreras, 2009, Jensen B. et A. Sawyer, 2013). En effet, bien que, d'une part, les résultats scolaires des enfants d'origine mexicaine aux ÉtatsUnis progressent, et que, d'autre part, les liquidités envoyées par les immigrés contribuent à l'amélioration des conditions d'éducation au Mexique, le croisement des données montre que, de part et d'autre de la frontière, l'expérience de la migration constitue un facteur majeur d'échec scolaire (Escobar Latapí A. et al., $2017: 29$ ).

21 Deux conférences binationales organisées en 2010 et 2016 par le Civil Rights Project de l'Université de Californie à Los Angeles, ont posé les bases d'une collaboration entre les universités et institutions éducatives d'un espace transfrontalier englobant les six comtés de la Californie du sud et la Basse-Californie (Mordechay, K. et C. Alfaro, 2019: 31). L'appellation «The Students We Share / Los Estudiantes que Compartimos » désigne désormais, et définit en tant que groupe sociologique distinct, l'ensemble des élèves d'origine mexicaine présents dans les systèmes éducatifs des deux pays et affectés ou susceptibles d'être affectés par une migration au cours de leur scolarité ${ }^{\text {. }}$

La forme la plus aboutie de cette collaboration est aujourd'hui le programme de formation des enseignants Formadores de Docentes binacionales qui vise, à terme, à substituer aux échanges de postes en place depuis les années 1980 dans le cadre du PROBEM, un programme de formation binational et bilingue. Ce programme repose sur un ensemble de modules de développement professionnel élaborés par les universités et les instituts de pédagogie engagés dans le projet ${ }^{10}$. Une des nouveautés est la mise en 
place de stages de formation accueillant des enseignants issus des établissements de Californie et de Basse-Californie et des formateurs des universités de San Diego et Tijuana. Les concepteurs du projet revendiquent en outre une identité transfrontalière forte, présentée comme un atout favorisant la compréhension et les échanges entre professionnels des deux pays (Gándara, P. et B. Jensen, 2021 : 60-62). La Basse-Californie prévoit de former, d'ici 2022, deux mille enseignants entièrement bilingues et sensibilisés à l'éducation interculturelle (Gándara, P., 2020 : 51).

S'agissant des programmes d'enseignement, les évolutions qui se profilent s'appuient sur des outils pédagogiques développés par les éducateurs mexicains pour l'éducation des hispanophones aux États-Unis. Par exemple, le programme SOL (Secondary On-Line Learning) est un cours de mathématiques et de sciences élaboré par l'Université de Guadalajara pour permettre aux élèves hispanophones scolarisés aux États-Unis de préparer les examens d'entrée à l'université. Depuis 2013, les contenus sont disponibles en espagnol et en anglais et alignés sur les normes nationales états-uniennes (common core standards) et mexicaines. Les conclusions du sommet Education without Borders / Educacion sin Fronteras organisé en juin 2017 dans le cadre du partenariat entre l'Université de Californie et les institutions mexicaines, UC-Mexico Initiative, prévoient l'utilisation de ces modules dans les établissements secondaires de Tijuana et de San Diego, ainsi que l'extension de la méthode à d'autres disciplines (UC-Mexico Initiative, 2017).

Enfin, le relais universitaire assuré par un réseau d'établissements regroupés au sein du Cali-Baja Education Consortium constitue une voie possible de poursuite d'études offrant des débouchés professionnels qui s'inscrivent dans la dynamique d'intégration économique de la région, notamment dans les domaines de l'aérospatiale et des équipements médicaux. Ce partenariat a vu le jour en 2017 et regroupe quatorze établissements d'enseignement supérieur de Californie et Basse-Californie ${ }^{11}$. Les programmes d'études sont conçus de manière à ce que les crédits obtenus puissent être reconnus par les établissements du réseau situés de part et d'autre de la frontière. Cette initiative entend contribuer à régler le problème des équivalences de diplômes auquel sont fréquemment confrontés les étudiants souhaitant poursuivre au Mexique un cursus universitaire débuté aux Etats-Unis. Le projet Enlace offre quant à lui aux lycéens des deux pays la possibilité de participer à différents programmes de recherche pendant l'été afin de préparer leur entrée à l'université.

\section{Conclusion}

Le durcissement des politiques migratoires aux États-Unis et la crise économique des années 2008-2009 ont influencé les déplacements des familles d'origine mexicaine et complexifié les trajectoires scolaires des enfants migrants internationaux. Les mécanismes de coopération éducative bilatérale mis en place par les gouvernements dans le contexte de migration sud-nord des années 1980 et 1990 s'avèrent aujourd'hui inadaptés aux évolutions démographiques, notamment s'agissant des élèves transnationaux issus du mouvement de migration de retour des années 2005-2015.

L'émergence de réseaux de coopération au niveau des États, dont la région transfrontalière de Californie et Basse-Californie constitue un exemple, montre que, dans un contexte politique qui laisse peu de place à la question de l'éducation des migrants internationaux, le rôle joué par les acteurs régionaux tend à se renforcer. En 
outre, les réseaux de collaboration déjà établis entre les institutions frontalières, en particulier les collaborations entre l'Université de Californie et les institutions mexicaines, constituent un terrain favorable pour le développement d'une approche transnationale des questions d'éducation et de migration qui ne se manifeste plus seulement par la délocalisation des programmes et des acteurs de l'enseignement audelà des frontières des pays, mais par le développement conjoint de programmes d'études et de formation binationaux et bilingues.

\section{BIBLIOGRAPHIE}

Anderson, Jill, « Bilingual, Bicultural, Not Yet, Binational: Undocumented Immigrant Youth in Mexico and the United States », Wilson Center, Mexico Institute, 2016, https://

www.wilsoncenter.org/sites/default/files/media/documents/publication/

bilingual_bicultural_not_yet_binational_undocumented_immigrant_youth_in_mexico_and_the_united_states.pdf, page consultée le 12 décembre 2020.

Borjian, Ali, Muñoz, Luz María de Cote, van Dijk, Sylvia, et al. « Transnational Children in Mexico: Context of Migration and Adaptation ", Diaspora, Indigenous, and Minority Education, vol. 10, $\mathrm{n}^{\circ} 1$, 2016, p. 42-54.

Cavazos, Lauro et Bartlett-Diaz, Manuel, « Memorandum of Understanding on Education, Nuevo Laredo, Mexico, August 17, 1990 », Washington, DC, U.S. Department of Education, 1990.

Center for U.S.-Mexican Studies, « The Students We Share: At the Border - San Diego \& Tijuana », 2017, https://usmex.ucsd.edu/_files/mmfrp_policy\%20brief_2017.pdf, page consultée le 12 décembre 2020.

Córdova Villalobos, José Angel et Arne Duncan, « Annex IX to the Memorandum of Understanding Between the Government of the United Mexican States and the Government of the United States of America », Washington, DC, U.S. Department of Education, 2012.

de la Garza, Rodolfo O., « Foreign Policy Comes Home: The Domestic Consequences of the Program for Mexican Communities Living in Foreign Countries », in de la Garza, Rodolfo O. et Jesus Velasco (dir.), Bridging the Border, Transforming U.S.-Mexico Relations, Lanham, MD, Rowman and Littlefield Publishers, p. 69-88.

Dolson, David P. et Villaseñor, Gildardo, « Migrant Education Binational Program », in Flores, Judith LeBlanc (dir.), Children of La Frontera: Binational Efforts to Serve Mexican Migrant and Immigrant Students, Washington, DC, Office of Educational Research and Improvement, 1996, p. 125-134.

Escobar Latapí, Agustín, Lindsay Lowell et Susan Martin, « Binational Dialogue on Mexican Migrants in the U.S. and Mexico ", Mac Arthur Foundation, 2017, http://www.cisan.unam.mx, page consultée le 12 décembre 2020.

Gándara, Patricia, « The Students We Share: Falling Through the Cracks on Both Sides of the USMexico Border ", Ethnic and Racial Studies, vol. 43, n 1, 2020, p. 38-59.

Gándara, Patricia et Frances Contreras, The Latino Education Crisis: The Consequences of Failed Social Policies, Cambridge, MA, Harvard University Press, 2009. 
Gándara, Patricia et Bryant Jensen (dir.), The Students We Share: Preparing U.S. and Mexican Educators for our Transnational Future, Albany, NY: SUNY Press, 2021.

Giorguli Silvia E. et Edith Y. Gutiérrez, « Niños y jóvenes en el contexto de la migración internacional entre México y Estados Unidos », Coyuntura Demográfica, vol. 1, 2011, p. 21-25.

González-Barrera, Ana, « More Mexicans Leaving than Coming to the U.S. », Washington, DC, Pew Research Center, 2015, https://www.pewresearch.org/hispanic/2015/11/19/more-mexicansleaving-than-coming-to-the-u-s/, page consultée le 20 janvier 2021.

Hazán, Miryam, « Understanding Return Migration to Mexico: Towards a Comprehensive Policy for the Reintegration of Returning Migrants », Working Paper 193, Center for Comparative Immigration Studies, septembre 2014, https://ccis.ucsd.edu/_files/wp193.pdf, page consultée le 12 janvier 2021.

Hernández-León, Rubén et Víctor Zúñiga, « Contemporary Return Migration from the United States to Mexico-Focus on Children, Youth, Schools and Families ", Mexican Studies/Estudios Mexicanos, vol. 32, $n^{\circ}$ 2, 2016, p.171-181.

Herrera, Martha Cecilia et Erika Cecilia Montoya Zavala, « Child Migrants Returning to Culiacán, Sinaloa, Mexico. A familial, Educational, and Binational Challenge ", Ánfora, vol. 26, n 46, 2019, http://www.redalyc.org/articulo.oa?id=357857620006, page consultée le 12 décembre 2020.

Imaz, Cécilia, « L'immigration au cœur de la relation Mexique-États-Unis », Politique Américaine, vol. $8, n^{\circ}$ 2, 2007, p. 31-46.

Instituto de los Mexicanos en el Exterior, « Gobierno de México amplía apoyos a personas migrantes ", 23 mars 2021, https://www.gob.mx/ime/articulos/gobierno-de-mexico-ampliaapoyos-a-personas-migrantes-267407?idiom=es, page consultée le 30 mars 2021.

Israel, Emma et Jeanne Batalova, « Mexican Immigrants in the United States », Washington, D.C., Migration Policy Institute, novembre 2020, https://www.migrationpolicy.org/article/mexicanimmigrants-united-states-2019, page consultée le 12 janvier 2021.

Jensen, Bryant, Rebeca Mejía Arauz et Rodrigo Aguilar Zepeda, « Equitable Teaching for Returnee Children in Mexico ", Sinéctica, n 48, 2017, p. 1-20.

Jensen, Bryant et Adam Sawyer (dir.), Regarding Educación: Mexican-American Schooling, Immigration, and Bi-national Improvement, New York, Teachers College Press, 2013.

Johnson, Hans, Cesar Alesi Perez et Marisol Cuellar Mejia, « Immigrants in California », Washington, DC, Public Policy Institute, 2021, https://www.ppic.org/wp-content/uploads/jtfimmigrants-in-california.pdf, page consultée le 12 janvier 2021.

Laglagaron, Laureen, « Protection through Integration: The Mexican Government's Efforts to Aid Migrants in the United States », Washington, DC, Migration Policy Institute, 2010, https:// www.migrationpolicy.org/research/protection-through-integration-mexican-governmentsefforts-aid-migrants-united-states, page consultée le 12 janvier 2021

Lakhani, Nina et Mónica Jacobo, « Uprooted in Mexico: the US Children 'Returned' to a Country They Barely Know », The Guardian, 13 juillet 2016, https://www.theguardian.com/world/2016/ jul/13/immigration-us-children-migrants-returned-to-mexico, page consultée le 3 janvier 2021.

Malgouyres, François, « Entre le global et le local : décentralisation et recentralisation éducative. Approche comparée de trois États fédéraux latino-américains ", Spirale, Revue de recherches en éducation, $\mathrm{n}^{\circ}$ 51, 2013, p. 83-96. 
Martin-Wenzl, Mary, « Bi-national Education Initiatives: A Brief History », in Bryant Jensen et Adam Sawyer (dir.), Regarding Educación: Mexican-American Schooling, Immigration, and Bi-national Improvement, New York, Teachers College Press, 2013, p. 278-298.

Martinez-Leon, Natalia, and Patrick H. Smith, « Educating for Bilingualism in Mexican Transnational Communities ", NABE Journal of Research and Practice, vol. 1, n 1, 2003, p. 138-148.

Massey, Douglas S., Jorge Durand et Nolan J. Malone, Beyond Smoke and Mirrors: Mexican Immigration in an Era of Economic Integration, New York, Russell Sage Foundation, 2002.

Mordechay, Kfir, et Cristina Alfaro, « The Binational Context of the Students We Share: What Educators on Both Sides of the Border Need to Know », Kappa Delta Pi Record, vol. 55, $\mathrm{n}^{\circ} 1$, January-March 2019, p. 30-35.

Osuna García, José Candelario et Jocelyne Rabelo Ramírez, « Estudiantes transnacionales y calidad de los sistemas educativos en la región Tijuana-San Diego », Revista Universidad en Diálogo, vol. 9, n. ${ }^{\circ}$ 1, Enero-Junio 2019, p. 63-85, https://www.revistas.una.ac.cr/index.php/dialogo/index\%0d, page consultée le 3 juillet 2021.

Panait, Catalina et Víctor Zúñiga, « Children Circulating between the U.S. and Mexico, Fractured Schooling and Linguistic Ruptures ", Mexican Studies/Estudios Mexicanos, vol. 32, n² 2, 2016, p. 226-251.

Passel, Jeffrey, D’Vera Cohn et Ana González-Barrera, « Net Migration from Mexico Falls to Zero and Perhaps Less », Washington D.C., Pew Research Center, 2012, https://www.pewresearch.org/ hispanic/2012/04/23/net-migration-from-mexico-falls-to-zero-and-perhaps-less/, page consultée le 12 mars 2021.

Portes, Alejandro, Luis E. Guarnizo et Patricia Landolt, « The Study of Transnationalism: Pitfalls and Promise of an Emergent Research Field », Ethnic and Racial Studies, vol. 22, n² 2 Mars 1999, p. 217-237.

Selee, Andrew, Vanishing Frontiers: The Forces Driving Mexico and the United States Together, New York: PublicAffairs, 2018.

Selee, Andrew, Silvia E. Giorguli-Saucedo, Ariel G. Ruiz Soto, et al., « Investing in the Neighborhood: Changing Mexico-US Migration Patterns and Opportunities for Sustainable Cooperation », Washington, DC, Migration Policy Institute, 2019.

Sierra Pérez, Susana Evelyn et Yara Amparo López López, « Infancia migrante y educación transnacional en la frontera México - Estados Unidos », Revista sobre la Infancia y la Adolescencia, $\mathrm{n}^{\circ}$ 4, Mars 2013, p. 28-54.

Smith, Michael P. et Luis Eduardo Guarnizo (dir.), Transnationalism from Below, New Brunswick, Transaction Publishers, 1998.

Tapia, Javier et Carla Dorn, « The Educational Convergence of the U.S. and Mexico: Challenges and Possibilities », Journal of International and Global Studies, vol. 10, n 1, 2019, p. 78-90.

UC-Mexico Initiative, « First Summit for Education without Borders: California-Mexico », 28 juin 2017, https://ucmexicoinitiative.ucr.edu/docs/2017\%20Educ\%20Summit_June\%2020171.pdf, page consultée le 22 février 2021.

U.S. Department of State, « U.S. Relations with Mexico », septembre 2020, www.state.gov/u-srelations-with-mexico, page consultée le 1er mars 2021.

Varisco de Garcia, Norma et Eugene E. Garcia, « Teachers for Mexican Migrant and Immigrant Students ", in Flores, Judith LeBlanc (Ed.), Children of La Frontera: Binational Efforts to Serve Mexican 
Migrant and Immigrant Students, Washington, DC: Office of Educational Research and Improvement, 1996, p. 153-163.

Zúñiga, Víctor, « Migration de retour : Un regard quantitatif sur les enfants arrivant au Mexique en provenance des États-Unis ", Hommes et Migrations, n 1296, 2012, p. 88-98.

Zúñiga, Víctor et Edmund T. Hamann, « Sojourners in Mexico with U.S. School Experience: A New Taxonomy for Transnational Students », Comparative Education Review, vol. 53, n 3, 2009, p. 329-353.

Zúñiga, Víctor et Edmund T. Hamann, « Understanding American Mexican Children », in Bryant Jensen et Adam Sawyer (dir.), Regarding Educación: Mexican-American Schooling, Immigration, and Binational Improvement, New York : Teachers College Press, 2013, p. 172-188.

Zúñiga, Víctor et Edmund T. Hamann, « De las escuelas de Estados Unidos a las escuelas de México: Desafíos de política educativa en el marco de la Gran Expulsión », Faculty Publications: Department of Teaching, Learning and Teacher Education, $\mathrm{n}^{\circ}$ 367, 2019.

Zúñiga, Víctor et Edmund T. Hamann, « Children's Voices about 'Return' Migration from the United States to Mexico: The 0.5 Generation », Children's Geographies, vol. 19, n 1, 2021, p. 88-100.

Zúñiga, Víctor, Edmund T. Hamann et Juan Sánchez García, « From Nuevo León to the USA and Back Again: Transnational Students in Mexico », Journal of Immigrant and Refugee Studies, vol. 6 , $\mathrm{n}^{\circ}$ 1, 2008a, p. 60-84.

Zúñiga, Víctor, Edmund T. Hamann et Juan Sánchez García, « Alumnos transnacionales: Las escuelas mexicanas frente a la globalización », Faculty Publications: Department of Teaching, Learning and Teacher Education, $\mathrm{n}^{\circ}$ 97, $2008 \mathrm{~b}$.

Zúñiga, Víctor et Maria Vivas-Romero, « Mexique : conséquences de la migration internationale sur l'éducation des enfants », Alternatives Sud, n² 22, 2015, p. 87-103.

\section{NOTES}

1. On estime à environ 30 millions le nombre de Mexicains entrés aux États-Unis entre 1965 et 1986 (28 millions y sont entrés illégalement, 1,3 millions, légalement, et 46000 en tant que travailleurs saisonniers). Au cours de la même période, 23,4 millions de Mexicains sans-papiers et 200000 immigrés légaux sont repartis vers le Mexique (Massey, D. et al., 2002 : 45).

2. Selon le Pew Research Center, en 2005, 60\% des immigrés mexicains vivant aux Etats-Unis y résidaient depuis plus de dix ans. Ce chiffre n'a cessé de progresser depuis pour atteindre $78 \%$ en 2017, date des dernières données disponibles (Pew Research Center, 2019).

3. Des enquêtes quantitatives ont été menées au Mexique dans les États de Nuevo León (2004), Zacatecas (2005), Puebla (2009) et Jalisco (2010). Les 54000 questionnaires diffusés ont permis de montrer qu'environ 3\% des élèves étaient "transnationaux », c'est-à-dire qu'ils avaient effectué une partie de leur scolarité aux Etats-Unis. Pour une présentation synthétique des résultats, voir Zúñiga V., 2012.

4. Dans cet article, nous reprenons la distinction faite par Víctor Zúñiga et Edmund T. Hamann et désignons comme «transnationaux » les élèves ayant fréquenté les systèmes scolaires des deux pays, indépendamment du pays de naissance de ces élèves. L'adjectif «binational » renverra quant à lui aux élèves bénéficiant de la double nationalité, états-unienne en vertu du droit du sol et mexicaine en vertu de celui du sang. 
5. Many of these children have spent most of their lives in the shadows in both the United States and Mexico - first as children of undocumented parents in the United States and later as children who cannot prove their identity and citizenship in Mexico. Traduction de l'auteure.

6. Esta población escolar no está contemplada en los planes y programas educativos de México, ni en el Plan nacional de desarrollo 2013-2018, ni en el Programa sectorial de educación 2013-2018, ni en el Modelo educativo 2016, documentos que dan sustento a la reforma educativa 2016. Traduction de l'auteure.

7. Es un espacio donde se encuentran estudiantes que han iniciado sus estudios en México pero son de nacimiento estadounidense y con alumnos que siempre han estudiado en Estados Unidos pero son mexicanos de nacimiento. Los niños migrantes desarrollan su experiencia y definen su identidad cruzando las fronteras que delimitan los Estados nacionales. Frente a esa frontera que divide, los migrantes transnacionales descubren otras acepciones posibles de comprender esa noción tan llena de significados. Traduction de l'auteure.

8. La moyenne nationale est de $1,6 \%$ en 2015.

9. Cette population se répartit comme suit : 600000 élèves transnationaux au Mexique, 500000 élèves mexicains scolarisés aux États-Unis et 7 millions d'enfants dont un des parents est immigré mexicain et dont les familles sont susceptibles de retourner vivre au Mexique. Voir Gándara, P., 2020.

10. Les principaux acteurs de cette collaboration sont le secrétariat à l'Éducation de BasseCalifornie, le département de l'Éducation de Californie, les Universités de Californie à San Diego et Los Angeles, l'Institut national de pédagogie du Mexique, Universidad Pedagógica Nacional, l'École normale de Tijuana, Normal Fronteriza et l'association de Californie pour l'enseignement bilingue, California Association for Bilingual Education.

11. Les institutions participantes sont les suivantes: UC San Diego, Universidad Nacional Autónoma de México, Universidad Autónoma de Baja California, Centro de Ensenanza Tecnica y Superior, Universidad Politécnica de Baja California, Universidad Tecnológica de Tijuana, Instituto Tecnológico de Tijuana, Instituto Tecnológico de Mexicali, Universidad de Tijuana, Centro Universitario 16 de Septiembre, Colegio La Paz, Instituto Altazor, Instituto Cumbres et Instituto Mexico.

\section{RÉSUMÉS}

La coopération entre le Mexique et les États-Unis dans le domaine de l'éducation est constituée d'un ensemble de programmes et d'accords opérés à plusieurs niveaux de gouvernance. À la croisée des questions de migration et de développement économique, cette collaboration a, depuis son origine, été centrée sur l'intégration des enfants d'origine mexicaine dans le système scolaire états-unien. Cet article se propose d'examiner l'histoire de cette coopération à la lumière des enjeux nouveaux posés par l'émergence d'une génération d'élèves transnationaux, issus d'un important mouvement de migration de retour vers le Mexique depuis 2005. Scolarisés alternativement dans l'un, puis l'autre pays, ces élèves représentent une part de plus en plus importante de la population scolaire du Mexique. Après avoir évoqué les évolutions et les limites des politiques éducatives fédérales, l'analyse se concentrera sur les initiatives prises au niveau régional et montrera que les collaborations nouvelles qui se font jour entre les institutions éducatives de Californie et de Basse-Californie ouvrent la voie à une transnationalisation de l'éducation favorisant la circulation des élèves d'origine mexicaine d'un système scolaire à l'autre. 
Cooperation between Mexico and the United States in the field of education consists of an array of programs and agreements operating at several levels of governance. At the crossroads of migration and economic issues, this collaboration has since its inception focused on the integration of children of Mexican descent into the U.S. school system. This article examines the history of this cooperation in light of the new challenges posed by the emergence of a generation of transnational students, the result of a major movement of return migration to Mexico since 2005. These students, who attend school alternately in one country and then the other, represent an increasingly important part of the Mexican school population. After discussing the evolution and limitation of federal educational policies, the analysis will show that the new collaborations that are emerging between the education institutions of California and Baja California pave the way for a transnationalization of education that facilitates the circulation of students of Mexican origin from one school system to the other.

La cooperación entre México y Estados Unidos en materia de educación se compone de una serie de programas y acuerdos que operan en varios niveles de gobierno. En la encrucijada de la migración y el desarrollo económico, esta colaboración se ha centrado desde sus inicios en la integración de los niños de ascendencia mexicana en el sistema escolar estadounidense. Este artículo examina la historia de esta cooperación a la luz de los nuevos retos que plantea la aparición de una generación de estudiantes transnacionales, resultado de un importante movimiento migratorio de retorno a México desde 2005. Estos alumnos, que van a la escuela alternativamente en un país y luego en el otro, representan una parte cada vez más importante de la población escolar de Mexico. Después de discutir los desarrollos y limitaciones de las políticas educativas federales, el análisis se centrará en las iniciativas tomadas a nivel regional y mostrará que las nuevas colaboraciones que están surgiendo entre las instituciones educativas de California y Baja California allanan el camino para una transnacionalización de la educación que favorece el movimiento de los estudiantes de origen mexicano de un sistema escolar a otro.

\section{INDEX}

Mots-clés : Éducation, coopération, transnationalisme, migration internationale, enfants migrants

Keywords : Education, cooperation, transnationalism, international migration, migrant children Palabras claves : Educación, coopération, transnacionalismo, migración internacional, niños migrantes

\section{AUTEUR}

\section{ISABELLE SINIC}

Maîtresse de conférences en études anglophones. Université Toulouse Capitole, 2 rue du Doyen Gabriel-Marty, 38000 Toulouse. Laboratoire Cultures Anglo-Saxonnes (CAS) - Université Toulouse Jean Jaurès Isabelle.sinic@ut-capitole.fr 